\title{
Short message service phone reminder as an important tool to reduce absenteeism for atendance at healthcare appointments
}

\author{
Mirian Farias, Daniel Klug, Balduino Tschiedel, Matilde Gerchman, Marcia Puñales* \\ From 20th Brazilian Diabetes Society Congress \\ Porto Alegre, Brazil. 11-18 November 2015
}

\section{Background}

Absenteeism, or missing scheduled appointments is a serious problem to the healthcare system, affecting healthcare institutions that cannot maximize their staff appointments, thus increasing their costs, and also to patients, who face difficulties in accessing the system.

\section{Objective}

The aim of this study was to assess whether sending scheduled appointment date reminders through Short Message Service (SMS) for attendance at a healthcare unit would reduce the absenteeism in our institution.

\section{Materials and methods}

The study enrolled patients who scheduled appointments with an interdisciplinary team (up to 5 scheduled appointments per shift), from January-December 2012, before the SMS reminders, and compared to January-December of 2013 and 2014. Absenteeism was analyzed by the total number of patients scheduled appointments (TSA), compared to the absentees at the appointed date and also the number of health care providers scheduled appointments (NSA) compared to the team's idle time during the study period. The SMS was sent a week before the appointment date.

\section{Results}

In 2012, before the SMS intervention, TSA absenteeism was $20.0 \pm 3.3 \%$ (7736 scheduled patients/1524 no-show patients), in 2013 was $15.9 \pm 2.1 \%(7509 / 1199)$ and in 2014 was $15.4 \pm 1.8 \%(7285 / 1118)$. A TSA absenteeism reduction of $20.5 \%(\mathrm{p}=0.0024)$ was observed in the first year of the intervention and of $23.0 \%$ (0.0004) in the second year. The NSA absenteeism in 2012 was $14.9 \pm 1.9 \%$ (17196 scheduled appointments/2510 idle time), in 2013 was $11.9 \pm 1.9 \%(16674 / 1988)$ and in 2014 was $11.5 \pm 1.7 \%$ (17867 /2024). During the study period, there was an idleness reduction of TSA (2012: $127 \pm 14.7,2013$ : $99.9 \pm 19.0$ and $2014: 93.2 \pm 17.9, \mathrm{p}=0.0003)$ and NSA (2012: 209.2 $\pm 26.6, \quad 2013: \quad 165.7 \pm 41.9$ and 2014: $168.7 \pm 31.4, \mathrm{p}=0.0052)$.

\section{Conclusion}

The use of phone reminders with scheduled appointment dates through SMS was effective in reducing healthcare absenteeism, being an important tool to optimize the services offered by the institution.

Published: 11 November 2015

\section{doi:10.1186/1758-5996-7-S1-A258}

Cite this article as: Farias et al:: Short message service phone reminder as an important tool to reduce absenteeism for atendance at healthcare appointments. Diabetology \& Metabolic Syndrome 2015 7(Suppl 1):A258.

\footnotetext{
* Correspondence: mpunales@aeromundi.com.br

Hospital da Criança Conceiçao e Instituto da Criança Com Diabetes-GHC, Porto Alegre, Brazil
} 